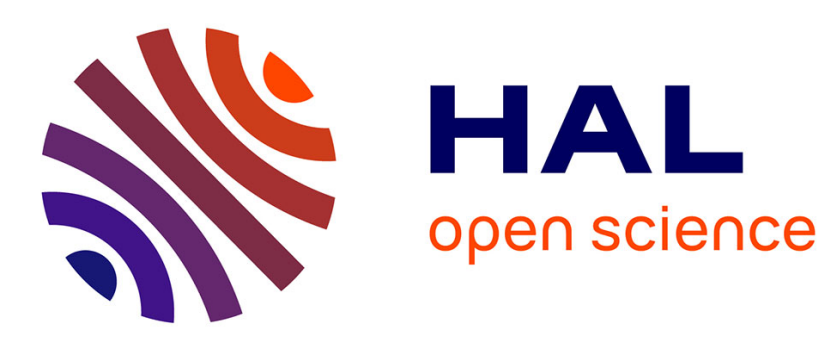

\title{
Stabilization of an Exploited Fish Population
}

Abderrahim Ouahbi, Abderrahman Iggidr, Mohamed El Bagdouri

\section{To cite this version:}

Abderrahim Ouahbi, Abderrahman Iggidr, Mohamed El Bagdouri. Stabilization of an Exploited Fish Population. Systems Analysis Modelling Simulation, 2003, 43 (4), pp.513-524. 10.1080/02329290290028543. hal-00867272

HAL Id: hal-00867272

https://hal.inria.fr/hal-00867272

Submitted on 27 Sep 2013

HAL is a multi-disciplinary open access archive for the deposit and dissemination of scientific research documents, whether they are published or not. The documents may come from teaching and research institutions in France or abroad, or from public or private research centers.
L'archive ouverte pluridisciplinaire HAL, est destinée au dépôt et à la diffusion de documents scientifiques de niveau recherche, publiés ou non, émanant des établissements d'enseignement et de recherche français ou étrangers, des laboratoires publics ou privés. 


\title{
Stabilization of an Exploited Fish
}

\section{Population *}

\author{
A.Ouahbi ${ }^{b} \quad$ A. Iggidr ${ }^{\ddagger} \quad$ M. El Bagdouri ${ }^{\dagger \dagger}$ \\ ${ }^{\ddagger}$ CONGE Project \\ INRIA Lorraine \& CNRS UPRESA 7035 \\ I.S.G.M.P. Bat. A, Ile du Saulcy \\ 57045 Metz cedex 01, FRANCE. \\ e-mail: iggidr@loria.fr

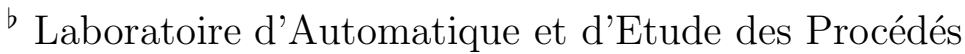 \\ Faculté des Sciences-Semlalia, Université Cadi Ayyad \\ B.P. 2390, Marrakesh, MOROCCO. \\ e-mail: elbagdouri@ucam.ac.ma
}

\begin{abstract}
The purpose of the paper is to design a fishing strategy in order to regulate an exploited fish population. To this end, we use a stagestructured model of a harvested fish population that has been derived in [5]. We give a formula for the fishing effort as a feedback control that allows to stabilize the system.
\end{abstract}

${ }^{*}$ travail réalisé avec la contribution financière du CNCPRST (Maroc) et du CNRS (France) dans le cadre du PICS $n^{\circ} 78$.

${ }^{\dagger}$ Corresponding author. 
Keys words: Continuous system, Feedback control, Population dynamics, Stabilization.

\section{Introduction}

The use of dynamic population models has become common in the study of exploited fish populations. These models are used to provide qualitative and quantitative descriptions of events in various fisheries, to give predictions of the future evolution of a given population and they are also useful for making policy decisions about fisheries.

In this paper we consider the following stage-structured model that has been built in [5]. It includes $(n+1)$ stages represented by their abundance $X_{i}(t)$, stage 0 being the pre-recuits stage. Each stage is characterized by its fecundity, mortality and predation rates (for more details, see [5]):

$$
\left\{\begin{aligned}
\dot{X}_{0}(t) & =-\alpha X_{0}(t)-m_{0} X_{0}(t)+\sum_{i=1}^{n} f_{i} l_{i} X_{i}(t)-\sum_{i=0}^{n} p_{i} X_{i}(t) X_{0}(t) \\
\dot{X}_{1}(t) & =\alpha X_{0}(t)-\alpha X_{1}(t)-m_{1} X_{1}(t) \\
\vdots & \\
\dot{X}_{n}(t) & =\alpha X_{n-1}(t)-\alpha X_{n}(t)-m_{n} X_{n}(t)
\end{aligned}\right.
$$

where

- $m_{i}$ : linear mortality rate.

- $\alpha$ : linear aging coefficient.

- $p_{0}$ : juvenile competition parameter.

- $p_{i}$ : predation rate of class $i$ on class 0 . 
- $f_{i}$ : fecundity rate of class $i$.

- $l_{i}$ : reproduction efficiency of class $i$.

As suggested in [5], the mortality coefficient can be written as a sum of the natural mortality rate $M_{i}$ and the fishing mortality coefficient $F_{i}$. Hence one can write:

$$
m_{i}=M_{i}+q_{i} E
$$

where $q_{i}$ is the catchability of stage $i$ and $E$ is the fishing effort that can be seen as a control term. The goal of this paper is to show how to control this system by acting on the fishing effort $E$. To this end, we rewrite it as follows:

$$
\begin{cases}\dot{X}_{0}(t) & =-\alpha_{0} X_{0}(t)+\sum_{i=1}^{n} f_{i} l_{i} X_{i}(t)-\sum_{i=0}^{n} p_{i} X_{i}(t) X_{0}(t) \\ \dot{X}_{1}(t) & =\alpha X_{0}(t)-\left(\alpha_{1}+q_{1} E(t)\right) X_{1}(t) \\ \vdots & \\ \dot{X}_{n}(t) & =\alpha X_{n}(t)-\left(\alpha_{n}+q_{n} E(t)\right) X_{n}(t)\end{cases}
$$

where

- $M_{i}$ is the natural mortality rate.

- $\alpha_{i}=\alpha+M_{i}$

- $q_{i}$ is the relative catchability coefficient of class $i$.

- $E$ is the fishing effort.

Our aim is to construct a feedback control $E=u(X)$ to ensure the survival of the population. More precisely, we shall compute $u(X)$ in such a way that 
the closed loop system (2) with this feedback has a nontrivial equilibrium state which is globally asymptotically stable.

In the following we shall use these notations:

$\mathbb{R}_{+}^{n+1}=\left\{X=\left(X_{0}, X_{1}, \ldots, X_{n}\right) \in \mathbb{R}^{n+1} \mid X_{i} \geq 0, i=0 \ldots n+1\right\}$.

$\Omega=\operatorname{Int}\left(\mathbb{R}_{+}^{n+1}\right)$ is the interior of $\mathbb{R}_{+}^{n+1}$, that is $\Omega=\left\{X \in \mathbb{R}^{n+1} \mid X_{i}>\right.$ $0, i=0 \ldots n+1\}$.

$X\left(t, X^{0}\right)$ is the solution of $(2)$ with initial state $X^{0}$, that is $X\left(0, X^{0}\right)=$ $X^{0}$.

\section{Main result}

\subsection{Stability with a constant fishing effort}

Consider the system with a constant fishing effort $\bar{E}$ :

$$
\left\{\begin{array}{l}
\dot{X}_{0}(t)=-\alpha_{0} X_{0}+\sum_{i=1}^{n} f_{i} l_{i} X_{i}(t)-\sum_{i=1}^{n} p_{i} X_{i}(t) X_{0}(t)-p_{0} X_{0}^{2}(t) \\
\dot{X}_{i}(t)=\alpha X_{i-1}(t)-\left(\alpha_{i}+q_{i} \bar{E}\right) X_{i}(t) \quad \text { for } i=1, \ldots, n
\end{array}\right.
$$

The origin is an equilibrium point which corresponds to an extinct population and is therefore not very interesting. Under the following nonlinearity and survival conditions ([5]):

(H1) $\quad \sum_{i=0}^{n} p_{i} \neq 0$

(H2) $\quad \sum_{i=1}^{n} f_{i} l_{i} \pi_{i}>\alpha_{0}$

where

$$
\pi_{i}=\frac{\alpha^{i}}{\prod_{j=1}^{i}\left(\alpha_{i}+q_{i} \bar{E}\right)}
$$


system (3) has an other equilibrium $X^{*}$

$$
\left\{\begin{array}{l}
X_{0}^{*}=\frac{\sum_{i=1}^{n} f_{i} l_{i} \pi_{i}-\alpha_{0}}{p_{0}+\sum_{i=1}^{n} p_{i} \pi_{i}} \\
X_{i}^{*}=\pi_{i} X_{0}^{*}
\end{array}\right.
$$

In ([6], pp 65-74), it has been shown that the positive orthant $\Omega=\{X \in$ $\left.\mathbb{R}^{n+1} \mid X_{i}>0, i=0 \ldots n+1\right\}$ is invariant and the following stability results for system (1) has been derived. It has been proved that if assumptions $\mathbf{H 1}$, H2 are satisfied and if moreover

(H3) $\quad f_{n} l_{n} \neq 0$

$$
X_{0}^{*}<\mu=\min _{i=1, \ldots, n}\left(\frac{f_{i} l_{i}}{p_{i}}\right) \quad \text { for } f_{i} l_{i} p_{i} \neq 0
$$

then:

(i) the origin is unstable.

(ii) there exists an invariant domain $\mathcal{D}=\prod_{i=1}^{n}\left[a_{i}, b_{i}\right]$, with $0<a_{i}<b_{i}$, such that the nontrivial equilibrium $X^{*}$ belongs to $\mathcal{D}, X^{*}$ is asymptotically stable and its attraction domain contains $\mathcal{D}$.

The proof of (i) uses the properties of positives matrices in order to show that the linearized system around the origin has a positive eigenvalue associated to a positive eigenvector. The proof of (ii) uses the properties of cooperative systems [4]. The numbers $a_{i}$ can be chosen as small as one needs but the numbers $b_{i}$ are bounded by some functions of the parameters $f_{i}, l_{i}$ and $\pi_{i}$ (see [6] for more details).

Here we shall prove that the attraction domain of $X^{*}$ is the whole positive orthant $\Omega$. Our proof uses the Lyapunov theory. The advantage of this 
method is that we get a global result and the Lyapunov function allows us to construct a variable fishing effort as a function of the state of the system and that stabilizes the population at its nontrivial equilibrium.

Proposition 2.1 Let $\bar{E}$ be any positive constant fishing effort. If assumptions (H1), (H2), (H3) and $\mathbf{( H 4 )}$ are satisfied then the state $X^{*}$ is a globally asymptotically stable equilibrium state for system (3) defined on $\Omega$.

Proof: Let us consider the following Lyapunov function

$$
V(X)=\frac{1}{2}\left(\left(X_{0}-X_{0}^{*}\right)^{2}+\sum_{i=1}^{n}\left(\frac{\sum_{j=i}^{n} k_{j} \pi_{j}}{\alpha_{i}+q_{i} \bar{E}}\right)\left(\frac{X_{i}-X_{i}^{*}}{\pi_{i}}\right)^{2}\right)
$$

where

$$
k_{i}=f_{i} l_{i}-p_{i} X_{0}^{*} \quad i=1, \ldots, n
$$

$V$ is a positive definite and proper function of $X$

- $V(X)>0$ for $X \neq X^{*}$, and $V\left(X^{*}\right)=0$

- $\lim _{\|X\| \rightarrow+\infty} V(X)=+\infty$

The derivative of $V$ along the solutions of system (3) is

$$
\begin{aligned}
\dot{V}(X)= & \left(-\alpha_{0} X_{0}+\sum_{i=1}^{n} f_{i} l_{i} X_{i}-\sum_{i=1}^{n} p_{i} X_{i} X_{0}-p_{0} X_{0}^{2}\right)\left(X_{0}-X_{0}^{*}\right) \\
& +\sum_{i=1}^{n}\left(\frac{\sum_{j=i}^{n} k_{j} \pi_{j}}{\alpha_{i}+q_{i} \bar{E}}\right)\left(\frac{\alpha X_{i-1}-\left(\alpha_{i}+q_{i} \bar{E}\right) X_{i}}{\pi_{i}}\right)\left(\frac{X_{i}-X_{i}^{*}}{\pi_{i}}\right)
\end{aligned}
$$

Since

$$
\alpha X_{i-1}^{*}-\left(\alpha_{i}+q_{i} \bar{E}\right) X_{i}^{*}=0 \text { for all } i=1, \ldots, n
$$


and

$$
-\alpha_{0} X_{0}^{*}+\sum_{i=1}^{n} f_{i} l_{i} X_{i}^{*}-\sum_{i=1}^{n} p_{i} X_{i}^{*} X_{0}^{*}-p_{0} X_{0}^{* 2}=0,
$$

we get

$$
\begin{aligned}
\dot{V}(X)= & \left(\sum_{i=0}^{n} k_{i}\left(X_{i}-X_{i}^{*}\right)-\sum_{i=0}^{n} p_{i}\left(X_{i}-X_{i}^{*}\right)\left(X_{0}-X_{0}^{*}\right)\right)\left(X_{0}-X_{0}^{*}\right) \\
& +\sum_{i=1}^{n}\left(\frac{\sum_{j=i}^{n} k_{j} \pi_{j}}{\alpha_{i}+q_{i} \bar{E}}\right)\left(\frac{\alpha\left(X_{i-1}-X_{i-1}^{*}\right)}{\pi_{i}}-\left(\alpha_{i}+q_{i} \bar{E}\right)\left(\frac{X_{i}-X_{i}^{*}}{\pi_{i}}\right)\right)
\end{aligned}
$$

where

$$
k_{0}=-\left(\alpha_{0}+p_{0}\left(X_{0}+X_{0}^{*}\right)+\sum_{i=1}^{n} p_{i} X_{i}^{*}\right)
$$

Thus

$$
\begin{aligned}
& \dot{V}(X)=\left(k_{0}-\sum_{i=1}^{n} p_{i}\left(X_{i}-X_{i}^{*}\right)\right)\left(X_{0}-X_{0}^{*}\right)^{2}+\sum_{i=1}^{n} k_{i}\left(X_{i}-X_{i}^{*}\right)\left(X_{0}-X_{0}^{*}\right) \\
& +\sum_{i=1}^{n} \sum_{j=i}^{n} k_{j} \pi_{j}\left(\frac{\alpha}{\alpha_{i}+q_{i} \bar{E}}\left(\frac{X_{i-1}-X_{i-1}^{*}}{\pi_{i}}\right)-\left(\frac{X_{i}-X_{i}^{*}}{\pi_{i}}\right)\right)\left(\frac{X_{i}-X_{i}^{*}}{\pi_{i}}\right)
\end{aligned}
$$

Taking into account that $\frac{\alpha}{\left(\alpha_{i}+q_{i} \bar{E}\right) \pi_{i}}=\frac{1}{\pi_{i-1}}$, and remarking that

$$
k_{0}-\sum_{i=1}^{n} p_{i}\left(X_{i}-X_{i}^{*}\right) \leq-\alpha_{0}-p_{0} X_{0}^{*}
$$

it follows

$$
\begin{aligned}
\dot{V}(X) \leq & -\left(\alpha_{0}+p_{0} X_{0}^{*}\right)\left(X_{0}-X_{0}^{*}\right)^{2}+\sum_{i=1}^{n} k_{i}\left(X_{i}-X_{i}^{*}\right)\left(X_{0}-X_{0}^{*}\right) \\
& +\sum_{i=1}^{n}\left[\left(\sum_{j=i}^{n} k_{j} \pi_{j}\right)\left(\left(\frac{X_{i-1}-X_{i-1}^{*}}{\pi_{i-1}}\right)\left(\frac{X_{i}-X_{i}^{*}}{\pi_{i}}\right)-\left(\frac{X_{i}-X_{i}^{*}}{\pi_{i}}\right)^{2}\right)\right]
\end{aligned}
$$


The inequality $a b \leq \frac{1}{2}\left(a^{2}+b^{2}\right)$ that holds for any pair of numbers $a$ and $b$ allows to write the inequalities $\left(\pi_{0}=1\right.$ by convention)

$$
\begin{aligned}
\dot{V}(X) \leq & -\left(\alpha_{0}+p_{0} X_{0}^{*}\right)\left(X_{0}-X_{0}^{*}\right)^{2}+\sum_{i=1}^{n} k_{i}\left(X_{i}-X_{i}^{*}\right)\left(X_{0}-X_{0}^{*}\right) \\
& +\sum_{i=1}^{n}\left[\sum_{j=i}^{n} k_{j} \pi_{j}\left(\frac{1}{2}\left(\frac{X_{i-1}-X_{i-1}^{*}}{\pi_{i-1}}\right)^{2}-\frac{1}{2}\left(\frac{X_{i}-X_{i}^{*}}{\pi_{i}}\right)^{2}\right)\right] \\
\leq & \left(-\alpha_{0}-p_{0} X_{0}^{*}+\frac{1}{2} \sum_{i=1}^{n} k_{i} \pi_{i}\right)\left(X_{0}-X_{0}^{*}\right)^{2} \\
& +\sum_{i=1}^{n} k_{i}\left(X_{i}-X_{i}^{*}\right)\left(X_{0}-X_{0}^{*}\right) \\
& +\frac{1}{2} \sum_{i=1}^{n-1} \sum_{j=i+1}^{n} k_{j} \pi_{j}\left(\frac{X_{i}-X_{i}^{*}}{\pi_{i}}\right)^{2}-\frac{1}{2} \sum_{i=1}^{n} \sum_{j=i}^{n} k_{j} \pi_{j}\left(\frac{X_{i}-X_{i}^{*}}{\pi_{i}}\right)^{2} \\
\leq & \left(-\alpha_{0}-p_{0} X_{0}^{*}+\frac{1}{2} \sum_{i=1}^{n} k_{i} \pi_{i}\right)\left(X_{0}-X_{0}^{*}\right)^{2} \\
& +\sum_{i=1}^{n} k_{i} \pi_{i}\left(\frac{X_{i}-X_{i}^{*}}{\pi_{i}}\right)\left(X_{0}-X_{0}^{*}\right)-\sum_{i=1}^{n} k_{i} \pi_{i}\left(\frac{X_{i}-X_{i}^{*}}{\pi_{i}}\right)^{2}
\end{aligned}
$$

¿From the expression (4) we check that

$$
-\alpha_{0}-p_{0} X_{0}^{*}+\frac{1}{2} \sum_{i=1}^{n} k_{i} \pi_{i}=-\frac{1}{2} \sum_{i=1}^{n} k_{i} \pi_{i} .
$$

Thus

$$
\begin{aligned}
\dot{V}(X) & \leq-\frac{1}{2} \sum_{i=1}^{n} k_{i} \pi_{i}\left(\left(X_{0}-X_{0}^{*}\right)^{2}+2\left(\frac{X_{i}-X_{i}^{*}}{\pi_{i}}\right)\left(X_{0}-X_{0}^{*}\right)-\left(\frac{X_{i}-X_{i}^{*}}{\pi_{i}}\right)^{2}\right) \\
& \leq-\frac{1}{2} \sum_{i=1}^{n} k_{i} \pi_{i}\left(\left(X_{0}-X_{0}^{*}\right)-\left(\frac{X_{i}-X_{i}^{*}}{\pi_{i}}\right)\right)^{2} \\
& \leq 0
\end{aligned}
$$


The equilibrium $X^{*}$ is then Lyapunov stable for system (3). Note that all solutions of the system are bounded because $V$ is proper and its derivative is non positive. It remains to show the attraction of the equilibrium. Let

$$
W=\left\{X \in \mathbb{R}_{+}^{n+1} \mid \dot{V}(X)=0\right\}
$$

According to LaSalle theorem [3], all the solutions starting in $\Omega$ tend to $L$ the largest invariant set contained in $W$. Let $X \in L$, we have

$$
\dot{V}(X)=0 \Leftrightarrow X_{0}-X_{0}^{*}=\frac{X_{1}-X_{1}^{*}}{\pi_{1}}=\ldots=\frac{X_{n}-X_{n}^{*}}{\pi_{n}}
$$

Substituting (5) in (7) and by using the equalities $\pi_{i}=\frac{\alpha}{\alpha_{i}+q_{i} \bar{E}} \pi_{i-1}$, it follows that

$$
\alpha X_{i-1}-\left(\alpha_{i}+q_{i} \bar{E}\right) X_{i}=0 \text { for all } i=1, \ldots, n \text {. }
$$

Which implies that for all $i=1, \ldots, n, \quad \dot{X}_{i}=0$ and

$$
X_{i}=\pi_{i} X_{0}
$$

Since $L$ is invariant, we get $\dot{X}_{0}=0$, so

$$
\dot{X}_{0}=-\alpha_{0} X_{0}+\sum_{i=1}^{n} f_{i} l_{i} \pi_{i}\left(\frac{X_{i}}{\pi_{i}}\right)-\sum_{i=1}^{n} p_{i} \pi_{i}\left(\frac{X_{i}}{\pi_{i}}\right) X_{0}-p_{0} X_{0}^{2}=0
$$


Exploiting equality (8), we deduce that

$$
\left(-\alpha_{0}+\sum_{i=1}^{n} f_{i} l_{i} \pi_{i}-\sum_{i=1}^{n} p_{i} \pi_{i} X_{0}-p_{0} X_{0}\right) X_{0}=0
$$

This implies that $X_{0}=0$ or $X_{0}=X_{0}^{*}$ and so, by (8) we deduce that $L \subset\left\{0, X^{*}\right\}$.

But the origin can not be an omega-limit point: suppose there exists a state $X^{0} \in \Omega$ such that the solution $X\left(t, X^{0}\right)$, issued from $X^{0}$, tends to the origin as t tends to $+\infty$, then by continuity of $V, V\left(X\left(t, X^{0}\right)\right) \longrightarrow V(0)$ as $t \longrightarrow+\infty$. Since $V$ is nonincreasing along the solutions of the system, we have

$$
V\left(X^{0}\right) \geq V\left(X\left(t, X^{0}\right)\right) \geq V(0), \forall t \geq 0
$$

On the other hand, we have

$$
\begin{aligned}
V(0)-V(X) & =\frac{1}{2}\left(X_{0}^{* 2}+\sum_{i=1}^{n}\left(\frac{\sum_{j=i}^{n} k_{j} \pi_{j}}{\alpha_{i}+q_{i} \bar{E}}\right)\left(\frac{X_{i}^{*}}{\pi_{i}}\right)^{2}\right) \\
& -\frac{1}{2}\left(\left(X_{0}-X_{0}^{*}\right)^{2}+\sum_{i=1}^{n}\left(\frac{\sum_{j=i}^{n} k_{j} \pi_{j}}{\alpha_{i}+q_{i} \bar{E}}\right)\left(\frac{X_{i}-X_{i}^{*}}{\pi_{i}}\right)^{2}\right) \\
& =\frac{1}{2}\left(X_{0}^{* 2}+\sum_{i=1}^{n} \gamma_{i} X_{i}^{* 2}-\left(X_{0}-X_{0}^{*}\right)^{2}-\sum_{i=1}^{n} \gamma_{i}\left(X_{i}-X_{i}^{*}\right)^{2}\right) \\
& =\frac{1}{2}\left(X_{0}\left(2 X_{0}^{*}-X_{0}\right)+\sum_{i=1}^{n} \gamma_{i} X_{i}\left(2 X_{i}^{*}-X_{i}\right)\right)
\end{aligned}
$$

where $\gamma_{i}=\frac{\sum_{j=i}^{n} k_{j} \pi_{j}}{\left(\alpha_{i}+q_{i} \bar{E}\right) \pi_{i}^{2}}$. Hence,

$V(0)>V(X)$ for all $X \in U=\left\{X \in \mathbb{R}_{+}^{n+1}: 0<X_{i}<2 X_{i}^{*}, i=0, \ldots, n\right\}$.

This is a contradiction to (9). So, we conclude that all the solutions tend to the nontrivial equilibrium $X^{*}$. 
Remark 2.1 A sufficient condition to get $X_{0}^{*}<\mu$ is

$$
\bar{E} \geq \frac{\alpha \sum_{i=i_{0}}^{n}\left(f_{i} l_{i}-p_{i} \mu\right)}{q_{i_{0}}\left(p_{0} \mu+\alpha_{0}-\frac{\alpha}{\alpha_{1}} \sum_{i=1}^{i_{0}-1}\left(f_{i} l_{i}-p_{i} \mu\right)\right)} .
$$

with $i_{0}=\min \left\{i \in\{1, \ldots n\} / q_{i} \neq 0\right\}$

In fact for $\bar{E} \geq \frac{\alpha \sum_{i=i_{0}}^{n}\left(f_{i} l_{i}-p_{i} \mu\right)}{q_{i_{0}}\left(p_{0} \mu+\alpha_{0}-\frac{\alpha}{\alpha_{1}} \sum_{i=1}^{i_{0}-1}\left(f_{i} l_{i}-p_{i} \mu\right)\right)}$. we have

$$
\left(p_{0} \mu+\alpha_{0}\right) \geq \frac{\alpha}{q_{i_{0}} \bar{E}} \sum_{i=i_{0}}^{n}\left(f_{i} l_{i}-p_{i} \mu\right)+\frac{\alpha}{\alpha_{1}} \sum_{i=1}^{i_{0}-1}\left(f_{i} l_{i}-p_{i} \mu\right)
$$

Taking into account that $\pi_{i} \leq \frac{\alpha}{q_{i_{0}} \bar{E}}$ for all $i=i_{0}, \ldots n$, and $\pi_{i} \leq \frac{\alpha}{\alpha_{1}}$ for all $i=1, \ldots i_{0}-1$, we get

$$
\left(p_{0} \mu+\alpha_{0}\right) \geq \sum_{i=1}^{n}\left(f_{i} l_{i}-p_{i} \mu\right) \pi_{i}
$$

hence

$$
\mu \geq \frac{\sum_{i=1}^{n} f_{i} l_{i} \pi_{i}-\alpha_{0}}{p_{0}+\sum_{i=1}^{n} p_{i} \pi_{i}}=X_{0}^{*}
$$

\subsection{Stabilization with a variable fishing effort}

Now we suppose that the fishing effort is time varying and we propose to construct it as a state feedback control that allows to stabilize the system around its nontrivial equilibrium. Thus we want to find a smooth function $u(x)$ in such a way that the state $X^{*}$ is a globally asymptotically stable 
equilibrium point for the closed-loop system (2) with $E(t)=\bar{E}+u(X(t))$ :

$$
\left\{\begin{array}{c}
\dot{X}_{0}=-\alpha_{0} X_{0}+\sum_{i=1}^{n} f_{i} l_{i} X_{i}-\sum_{i=0}^{n} p_{i} X_{i} X_{0} \\
\dot{X}_{1}=\alpha X_{0}-\left(\alpha_{1}+q_{1} \bar{E}\right) X_{1}-q_{1} u X_{1} \\
\vdots \\
\dot{X}_{n}=\alpha X_{n}-\left(\alpha_{n}+q_{n} \bar{E}\right) X_{n}-q_{n} u X_{n}
\end{array}\right.
$$

In a condensed form this system can be written:

$$
\dot{X}=F(X)+u G(X)
$$

where

$$
F(X)=\left(\begin{array}{c}
-\alpha_{0} X_{0}+\sum_{i=1}^{n} f_{i} l_{i} X_{i}-\sum_{i=0}^{n} p_{i} X_{i} X_{0} \\
\alpha X_{0}-\left(\alpha_{1}+q_{1} \bar{E}\right) X_{1} \\
\vdots \\
\alpha X_{n}-\left(\alpha_{n}+q_{n} \bar{E}\right) X_{n}
\end{array}\right), \quad G(X)=\left(\begin{array}{c}
0 \\
-q_{1} X_{1} \\
\vdots \\
-q_{n} X_{n}
\end{array}\right)
$$

To stabilize this system we shall use and adapt the Jurdjevic-Quinn [1] stabilization procedure:

A candidate stabilizer is $u=\Phi(X)=-\langle G(X), \nabla V(X)\rangle$ where $\langle.,$.$\rangle is a$ scalar product on $\mathbb{R}^{n+1}$ and

$$
\nabla V(X)=\left(\begin{array}{c}
\frac{\partial V}{\partial X_{0}} \\
\frac{\partial V}{\partial X_{1}} \\
\vdots \\
\frac{\partial V}{\partial X_{n}}
\end{array}\right)
$$


Using the Lyapunov function introduced above, we get:

$$
\Phi(X)=\sum_{i=1}^{n} \gamma_{i} q_{i} X_{i}\left(X_{i}-X_{i}^{*}\right)
$$

Where

$$
\gamma_{i}=\frac{\sum_{j=i}^{n} k_{j} \pi_{j}}{\left(\alpha_{i}+q_{i} \bar{E}\right) \pi_{i}^{2}} .
$$

The derivative of $V$ along the trajectories of the closed loop system is:

$$
\begin{aligned}
\dot{V} & =\langle F(X), \nabla V(X)\rangle+u(X)\langle G(X), \nabla V(X)\rangle \\
& =\langle F(X), \nabla V(X)\rangle-\Phi(X)^{2} \\
& \leq-\frac{1}{2} \sum_{i=1}^{n} k_{i} \pi_{i}\left(\left(X_{0}-X_{0}^{*}\right)-\left(\frac{X_{i}-X_{i}^{*}}{\pi_{i}}\right)\right)^{2}-\Phi(X)^{2} \\
& \leq 0
\end{aligned}
$$

As in section 2.1, LaSalle Invariance Principle allows to conclude that $X_{0}^{*}$ is globally asymptotically stable.

However, the function $\Phi$ is unbounded and takes positive as well as negative values, hence $E=\bar{E}+u(X)$ can take negative values which has no sense ( $E$ is a fishing effort). Thus, instead of using the feedback given by the formula (12), we use the following bounded function:

$$
u(X)=\frac{2 \Phi(X)}{1+\Phi^{2}(X)} \bar{E}
$$

We then have $|u(X)| \leq \bar{E}$ which ensures that $0 \leq E(X) \leq 2 \bar{E}$ for all $X \in \Omega$. 


\section{Remarks and simulations}

1. The results of $[5,6]$ ensures the attraction of the equilibrium only for initial states that belong to $D=\prod_{i=0}^{n}\left[a_{i}, b_{i}\right]$. Our result proves that the attraction domain is the whole positive orthant. Here we give a simulation corresponding to a harvested fish population characterized by the parameter values given in table1, which are checked on from [5].

\begin{tabular}{|c|c|c|c|c|c|}
\hline Stage $i:$ & 0 & 1 & 2 & 3 & 4 \\
\hline \hline$p_{i}$ & 0.2 & 0 & 0.1 & 0.1 & 0.1 \\
\hline$f_{i}$ & & 0 & 0.5 & 0.5 & 0.5 \\
\hline$l_{i}$ & & 0 & 10 & 20 & 15 \\
\hline$m_{i}$ & 0.5 & 0.2 & 0.2 & 0.2 & 0.2 \\
\hline$q_{i}$ & 0 & 0 & 0 & 0.1 & 0.15 \\
\hline$M_{i}$ & 0.5 & 0.2 & 0.2 & 0.1 & 0.05 \\
\hline$\alpha$ & \multicolumn{5}{|c|}{0.8} \\
\hline$\alpha_{i}$ & 1.3 & 1 & 1 & 0.9 & 0.85 \\
\hline $\bar{E}$ & \multicolumn{5}{|c|}{1} \\
\hline$\alpha_{i}+q_{i} \bar{E}$ & 1.3 & 1 & 1 & 1 & 1 \\
\hline
\end{tabular}

Table1: The parameter values of a harvested fish population.

It is clear that this parameter values satisfy hypotheses (H1), (H2), (H3) and (H4). For these parameters we have $b_{0}=50, b_{1}=40, b_{2}=$ $32, b_{3}=17.06, b_{4}=10.9$. For the initial state $X_{\text {init }}=(100,80,15,20,9)$ which is outside the domain $D$, we obtain the following curves that confirm our results. 

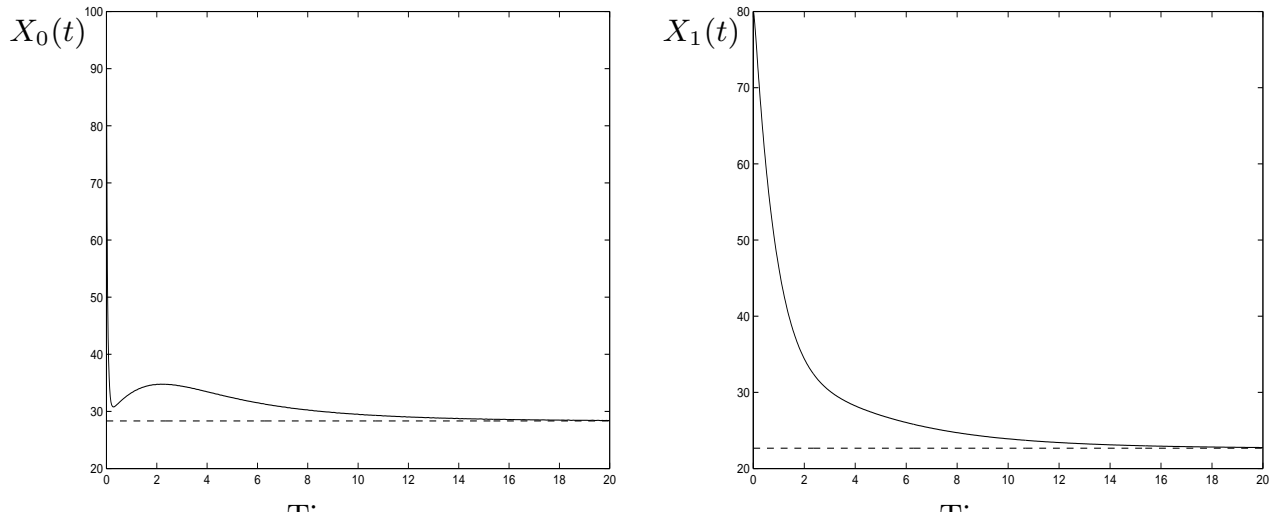

Time
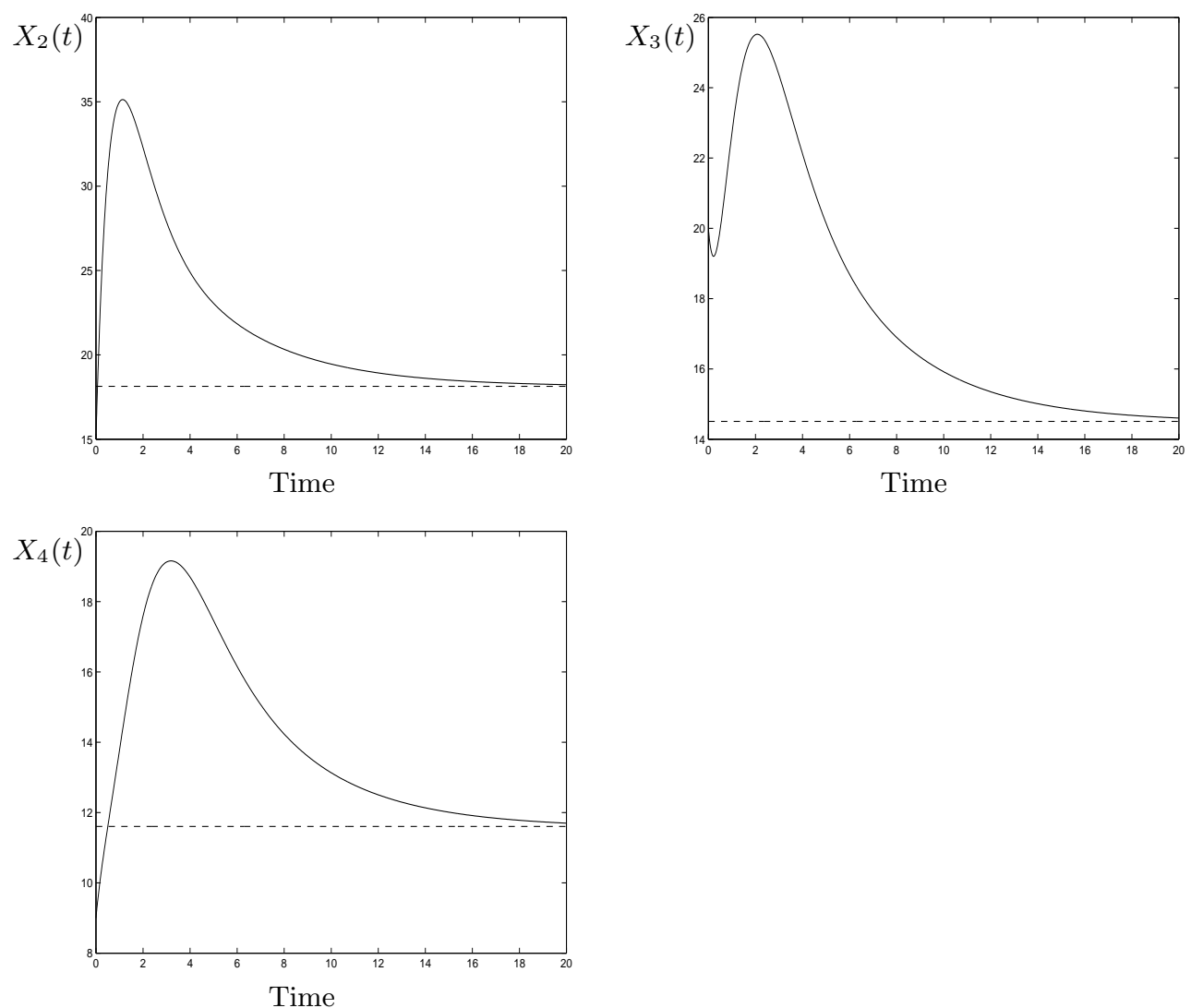
2. Our proof and the one of $[5,6]$ use the condition $X_{0}^{*}<\mu$. But we think that this condition is not necessary as it is suggested by the following example:

\begin{tabular}{|c|c|c|c|c|c|}
\hline Stage $i:$ & 0 & 1 & 2 & 3 & 4 \\
\hline \hline$p_{i}$ & 0.2 & 0 & 0.1 & 0.1 & 0.8 \\
\hline$f_{i}$ & & 0 & 0.5 & 0.5 & 0.5 \\
\hline$l_{i}$ & & 0 & 10 & 20 & 15 \\
\hline$m_{i}$ & 0.5 & 0.2 & 0.2 & 0.2 & 0.2 \\
\hline$q_{i}$ & 0 & 0.1 & 0.25 & 0.25 & 0.5 \\
\hline$M_{i}$ & 0.5 & 0.2 & 0.2 & 0.1 & 0.05 \\
\hline$\alpha$ & \multicolumn{7}{|c|}{0.8} & \\
\hline$\alpha_{i}$ & 1.3 & 0.98 & 0.95 & 0.95 & 0.9 \\
\hline $\bar{E}$ & \multicolumn{7}{|c|}{0.2} \\
\hline$\alpha_{i}+q_{i} \bar{E}$ & 1.3 & 1 & 1 & 1 & 1 \\
\hline
\end{tabular}

For these parameters $X_{0}^{*}=15.2495, \mu=9.375$. We have $X_{0}^{*}>\mu$. However the simulations show the attraction of the equilibrium $X^{*}$.
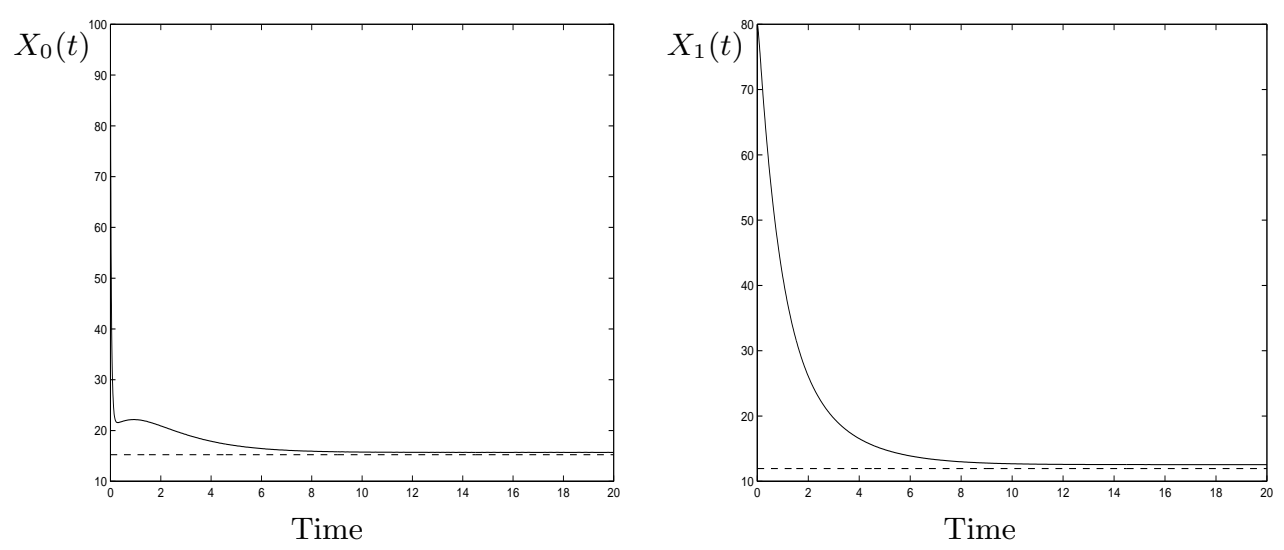

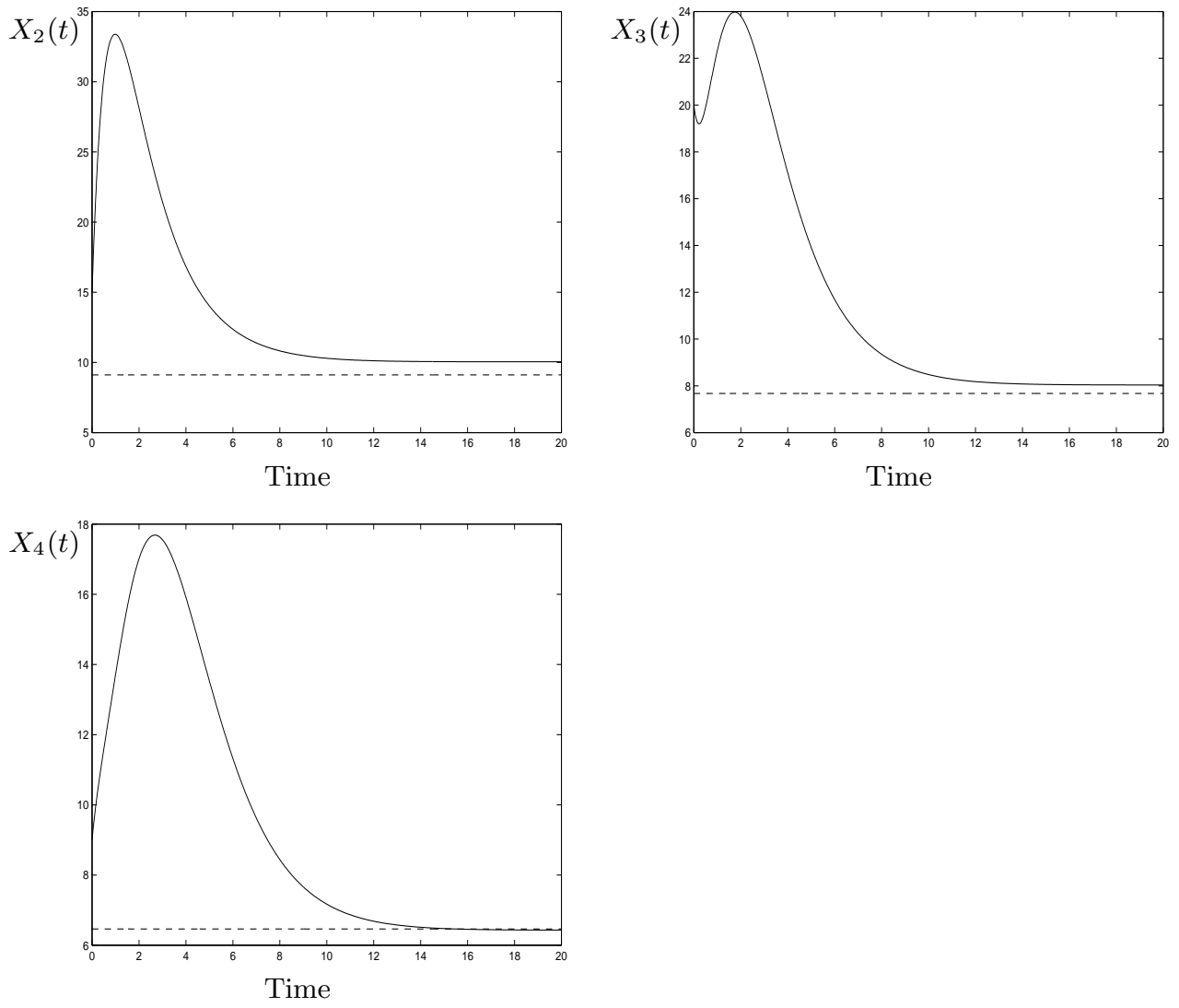

\section{References}

[1] V. Jurdjevic and J.P. Quinn. Controllability and stability. J. Diff. Equ., 28:381-389, 1978.

[2] H. Khalil. Nonlinear systems. Mcmillan, 1996.

[3] J.P. LaSalle and S. Lefschetz. Stability by Liapunov's direct method with applications. Academic Press, New-York, 1961. 
[4] H.L. Smith. Systems of ordinary differential equations which generate an order preserving flow. A survey result. SIAM Review, 30 (1):87-113, 1988.

[5] S. Touzeau and J.-L. Gouz. On the stock-recruitment relationships in fish population models. Environmental modelling and assessment, 3:87-93, 1998.

[6] S. Touzeau. Modèles de contrôle en gestion des pêches. Doctorate Thesis, University of Nice-Sophia Antipolis, France, 1997. 